\title{
A Framework for the Internationalization of Data-Intensive Web Applications
}

\author{
Alberto Belussi and Roberto Posenato \\ Dipartimento di Informatica - Università degli Studi di Verona \\ Ca' Vignal, Strada le Grazie, 15 \\ 37134 Verona, Italy \\ \{alberto.belussi, roberto.posenato\}@univr.it
}

\begin{abstract}
The globalization process makes it necessary to provide information in different languages in order to enlarge the audience of the information presented through the web. Therefore, it is often required to extend the web system for handling information in more than one language. This process is called internationalization of a system. An important requirement of this process is that it should be applied without a strong reorganization of the web application that implements the web site. We propose a general framework for the internationalization of data-intensive web applications based on the MVC-2 paradigm and a relational database system. This framework has been applied succesfully at the University of Verona (Italy) for the internationalization of the web sites of all departments and all faculties.
\end{abstract}

\section{Introduction}

In the last few years the globalization process has influenced the activities of any kind of organization around the world. One effect of this process concerns the production of information in more than one language. In general we call the adaption of an application to support a multilingual environment internationalization of an application, while the process of adaption of an application for a specific region or language, by adding local-specific components and translating text, is called localization of an application.

The internationalization of a web application can be a hard task, in particular if the application architecture does not conform to a standard schema, like the Model View Control (MVC) paradigm, or does not separate at least the logic and the presentation in different modules.

In this paper we propose a general framework for the internationalization of data-intensive web applications 65. based on the MVC-2 paradigm [1] and one or more relational database systems. This framework can also be applied to existing web applications with a low impact on code restructuring as shown by the experience at the University of Verona.

Previous works on data-intensive web applications regards mainly the definition of a design methodology and the implementation of case tools [7/5[4]6. Many other papers in the human-computer interaction area face the general problem 
of internationalization and localization of data presentation in web sites, considering in particular aspects like symbols or color perception in different cultures, format conventions (i.e., date presentation), etc. 2. To our knowledge, only in [8] a general approach for the internationalization of a web application including database schema extension is presented. In our approach, we extend the solution proposed in [8] by adding a general mechanism for queries specification and query results handling.

The paper is organized as follows. First, the problem of internationalization of a web application is described. Next, the proposed framework is presented by describing in details its components.

\section{Problem Definition and Proposed Solution}

In this paper with the term "web application" we refer to any software application that is accessible using a web browser. The internationalization of web applications regards the implementation of a mechanism that is able to produce, for each page the web application generates (master page), a set of pages with the same content but each one in a different language (translated pages). This problem can have different solutions with respect to the architecture of the web application and the level of update between the master pages and the translated ones that we intend to maintain.

According to the architecture, different solutions of the internationalization problem can be applied. In particular, in Static Web Applications the replication of the static HTML files with the translation of their content in the languages of interest is the widely used solution; of course, the maintenance of the consistency between the different replications can become a time consuming work 3]. In Data-Intensive Web Applications the replication is still a chance but it requires to generate one or more copies of the whole system. An alternative solution is to make the application language independent. This can be achieved by a revision of the whole application consisting of the following three steps: (1) Database extension: the database has to be extended in order to store the translations of data that are subject to change with respect to the language; (2) Queries rewriting: the queries have to be rewritten to take into account the new tables containing data translations; (3) Static text translation: the static text inside script files (like JSP, ASP, etc.) has to be replaced by a call to a procedure that computes the right translation according to the required language.

We propose a framework for making a data-intensive web application language independent. In particular, the framework can be applied to applications adopting the MVC-2 paradigm [1] in order to obtain the following results: (1) it should make the application language independent in the sense that the application should work with a certain set of foreign languages and this set can be extended without requiring any code modification; (2) it should allow one to extend the set of attributes subject to translations without requiring any code modification; (3) it should require the minimal database extension, but, at the same time, the minimal effort in query rewriting and the minimal performance worsening in query processing. 
Hereby we describe in details the proposed frameworks considering: database extension, queries rewriting and internationalization of state objects.

\subsection{Database Extension and Queries Rewriting}

A data-intensive web application interacts with one or more database servers, which provide dynamically the data to be published. This approach always guarantees web pages with up to date content. The internationalization process requires an extension of the database schema in order to store the translations of the information stored in the database.

We suggest the following general approach to extend the database schema, by supposing to adopt the Entity-Relationship model for the conceptual database design and a relational system for the database implementation:

1. For each entity type $E$ with attributes $\left\{A_{1}, \ldots, A_{n}\right\}$ having some attributes $\left\{A_{i_{1}}, \ldots, A_{i_{m}}\right\}$ subject to translation, we specify a new entity type $E_{\text {trans }}$, weak entity of $E$, with the following attributes $\left\{\right.$ language, $\left.A_{i_{1}}, \ldots, A_{i_{m}}\right\}$. This implies the creation of a new table $E_{\text {trans }}\left(K_{E}\right.$, language, $\left.A_{i_{1}}, \ldots, A_{i_{m}}\right)$ where $K_{E}$ is the set of attributes representing the primary key of $E$.

2. For each relationship type $R$ among entity types $\left\{E_{1}, \ldots, E_{n}\right\}$ with attributes $\left\{A_{1}, \ldots, A_{n}\right\}$ having some attributes $\left\{A_{i_{1}}, \ldots, A_{i_{m}}\right\}$ subject to translation, we replace $R$ with a new entity type $E_{-} R$, weak entity of $\left\{E_{1}, \ldots, E_{n}\right\}$, with the attributes $\left\{A_{1}, \ldots, A_{n}\right\}$ and we add a second entity type $E_{-} R_{\text {trans }}$, weak entity of $E_{-} R$, with the following attributes $\left\{\right.$ language, $\left.A_{i_{1}}, \ldots, A_{i_{m}}\right\}$. This implies the creation of a new table $R_{\text {trans }}\left(K_{R}\right.$, language, $\left.A_{i_{1}}, \ldots, A_{i_{m}}\right)$ where $K_{R}$ is the set of attributes representing the primary key of table $R$ ).

Regarding database schema extension other solutions could possibly be considered as discussed in [3]. After database extension, a query rewriting is necessary in order to include the translated attributes in the result of each query. The properties of this rewriting technique are the following ones: (1) it preserves the result cardinality that the query had before the internationalization; (2) it uses only two languages $L_{1}$ and $L_{2}$ as query parameters (these two languages are obtained by applying a custom rule to the preference languages list coming from the HTTP request, see [3] for details); (3) it extends each query result schema by applying the following rule: for each attribute $A$ subject to translation, we replace $A$ with three attributes $L 0-A, L 1-A$ and $L 2-A$, containing its values in $L_{0}$ (master language) and its translations in $L_{1}$ and $L_{2}$ respectively.

Given a generic query, SELECT $E_{1} \cdot A_{1}, \ldots, E_{k} \cdot A_{n}$ FROM $E_{1}, \ldots, E_{m}$ WHERE $C$, suppose that the table $E_{1}$ has a translation table $E_{1 \text { trans }}\left(K\right.$, language, $\left.A_{1}\right)$, then the query must be rewritten as follows:

SELECT $E_{1} \cdot A_{1} \mathrm{AS}$ " $L 0-A_{1}^{\prime \prime}, L 1 . A_{1} \mathrm{AS}$ " $L 1-A_{1}^{\prime \prime}, L 2 . A_{1} \mathrm{AS}$ " $L 2-A_{1}^{\prime \prime}, \ldots, E_{k} . A_{n}$ FROM $E_{1}$ LEFT JOIN $E_{1 \text { trans }}$ AS $L 1$ ON $E_{1} \cdot K=L 1 . K$

LEFT JOIN $E_{1 \text { trans }}$ AS $L 2$ ON $E_{1} \cdot K=L 2 . K, \ldots, E_{m}$

WHERE $C$ AND L1.language $=$ ? AND L2.language $=$ ?

Notice that, after the rewriting process, the resulting query contains some 
parameters, represented as "?", which stand for the requested language $L_{1}$ and the alternative language $L_{2}$ respectively.

This rewriting technique can be trivially extended to the general case in which table $E_{1}$ trans contains more than one attribute and there are other tables $E_{i}$ having a corresponding translation table $E_{i}$ trans. Moreover, given a query containing $m$ tables with attributes subject to translation, the number of outer joins that have to be added to the query is equal to $2 \mathrm{~m}$.

\subsection{Internationalization of State Objects}

In a MVC-2 data-intensive web application, state objects represent both the request from the browser and the data (often given by tuples of queries results) needed to build the response. Given the goal of applying language independent extensions, the more suitable way to do the task is to insert, for each object property $A$ subject to translation, a new property $A$-lang that will contain the language code of the value stored in $A$. In this way a state object can store values in any language with a minimal impact on its type restructuring.

Given a tuple $t$ of a query result $Q$, having translated values stored in the same tuple, a state object $O$ is created and is populated by applying the following approach: (1) for each attribute $A$ of $Q$ subject to translation choose one translated value in $t$ according to a custom rule; (2) put the chosen value in the corresponding property $A_{i}$ of $O$ and its language-code into property $A_{i}$-lang; (3) finally, put the other values belonging to attributes not subject to translation in the corresponding properties of $O$.

Therefore, each state objects constructor having a query result tuple as parameter, have to be modified according to the new proposed approach. Nevertheless, this process can be avoided if all constructors based on query result tuple are replaced with a single meta-method Populate. Method Populate works as follow: given a state object, a query result tuple, the requested language $L_{1}$ and one ore more alternative languages, it fills the state object with tuple values according to $L_{1}$ and the alternative languages. Populate can be implement under the following conditions: (1) the query result is written as described in Section 2.1; (2) the state object representing a tuple of a query result $Q$ contains at least as many properties as the attributes of $Q$ where each property has the same name of the corresponding attribute of $Q$ and contains a property for the language-code $A_{i^{-}}$ lang for each property $A_{i}$ subject to translation; (3) the programming language provides introspection mechanism for the state object and for the query result object as Java language provides with Reflection/Introspection mechanism. The pseudo-code of populate is shown in [3].

\section{The Experience at University of Verona}

The proposed approach has been applied for the internationalization of the web sites of the faculties and departments of the University of Verona (UNIVR). The UNIVR web system is organized as follows: (1) a portal site composed of static HTML files; (2) 8 faculties sites, each generated by a data-intensive web 
application (Faculty On Line - FOL); (3) 24 departments sites, each generated by a data-intensive web application (Department On Line - DOL). Both FOL and DOL are developed according to the MVC-2 paradigm using Servlets and JavaServer Pages technology; all the data come from one database containing the information about the teaching and research activities of the whole University. We have applied the proposed framework for the internationalization of FOL and DOL during 2003 and now the faculties and departments sites can show pages in four foreign languages (English, French, German and Spanish) and Italian. The impact of the internationalization process of the UNIVR web system can be summarized as follows: the database schema, that contained 275 tables, now contains 340 tables and the Java classes of FOL (DOL) contain 117 SQL queries as before the internationalization and they contain about $5 \%$ code lines more.

\section{Conclusions}

In this paper we have illustrated a framework for the internationalization of a data-intensive web application. The proposed solution has been studied for an application based on the MVC-2 paradigm and interacting with a relational database system. The framework was successfully applied for the internationalization of the web sites of University of Verona.

\section{References}

1. D. Alur, J. Cruspi, and D. Malks. Core J2EE Patterns: Best Practices and Design Strategies. Prentice Hall, 2001.

2. N. Aykin. Internationalization and Localization of the Web Sites. In Hans-Jörg Bullinger and Jürgen Ziegler, editors, Proceedings of HCI International '99, pages 1218-1222, Munich, Germany, August 22-26 1999.

3. Alberto Belussi and Roberto Posenato. Internationalizing Data-Intensive Web Applications. Technical Report DI 16/2004, Dip. di Informatica-Università di Verona, 2004.

4. S. Ceri, P. Fraternali, A. Bongio, S. Butti, R. Acerbis, M. Tagliasacchi, G. Toffetti, C. Conserva, R. Elli, F. Ciapessoni, and C. Greppi. Architectural Issues and Solutions in the Development of Data-Intensive Web Applications. In Proc. 1st Biennial Conf. on Innovative Data Systems Research, Asilomar, CA, USA, January 5-8 2003.

5. S. Ceri, P. Fraternali, and S. Paraboschi. Design Principles for Data-Intensive Web Sites. SIGMOD Record, 28(1):84-89, 1999.

6. P. Fraternali. Tools and Approaches for Developing Data-Intensive Web Applications: A Survey. ACM Computing Surveys, 31(3):227-263, 1999.

7. P. Merialdo, P. Atzeni, and G. Mecca. Design and Development of Data-Intensive Web Sites: The Anareus Approach. ACM Transactions on Internet Technology, 3(1):49-92, February 2003.

8. Inderjeet Singh, Beth Stearns, Mark Johnson, and the Enterprise Team. Design Enterprise Applications with the J2EE ${ }^{T M}$ Platform. Addison-Wesley Publishing Company, second edition, 2002. 